Int. J. Odontostomat.,

15(3):773-781, 2021.

\title{
Irrigación Activada por Láser en Endodoncia
}

\author{
Laser Activated Irrigation in Endodontics
}

Pablo Betancourt'; Josep Arnabat-Domínguez² \& Miguel Viñas ${ }^{3}$

BETANCOURT, P.; ARNABAT-DOMínGUEZ, J. \& VIÑAS, M. Irrigación activada por láser en endodoncia. Int. J. Odontostomat., 15(3):773-781, 2021.

RESUMEN: El objetivo de la endodoncia es prevenir o curar la periodontitis apical. Por lo tanto, los microorganismos que han colonizado el sistema de canales radiculares deben ser eliminados para promover la regeneración y cicatrización. Desafortunadamente, la existencia de canales accesorios, anastomosis, istmos, así como ramificaciones apicales, genera una compleja red tridimensional en el interior de la raíz, lo que dificulta la eliminación total de bacterias y detritus. La terapia endodóntica convencional utilizada actualmente presenta una serie de limitaciones.Se ha demostrado que los instrumentos endodónticos dejan un $35 \%$ o más de superficie dentinaria sin tratar. Por otro lado, la imposibilidad de generar un flujo turbulento en el interior de los canales, impide que el irrigante llegue a zonas de difícil acceso. Esto facilita la persistencia de biopelículas bacterianas y la supervivencia de un número importante de bacterias viables. La irrigación activada por láser (LAI) ha sido propuesta como una tecnología co-adyuvante a la terapia quimio-mecánica para optimizarla limpieza y desinfección. Los láseresde Er,Cr:YSGG (2780nm) y Er:YAG (2940nm) son los más utilizados. Estas dos longitudes de onda son capaces de ser absorbidas ampliamente por diferentes soluciones irrigantes, teniendo en cuenta un uso seguro y respetando los parámetros clínicos aceptados. La absorción de la energía del láser por parte de los irrigantes, genera burbujas de vapor en el interior del fluido, que liberan fuerzas de cizalla al implosionar. Este fenómeno, denominado cavitación, genera mayor limpieza y desinfección del interior del sistema de canales radiculares, incluso en zonas de difícil acceso. Diversos estudios microbiológicos y microscópicos, han demostrado la efectividad antibacteriana de LAI. Recientemente, se ha publicado que LAI tendría la capacidad de incrementarla capacidad antibacteriana del hipoclorito de sodio a baja concentración (un buen desinfectante pero extraordinariamente tóxico), lo que permitiría trabajar con concentraciones menores y por lo tanto más seguras para el paciente.

PALABRAS CLAVE: láser, irrigación, endodoncia, hipoclorito de sodio, cavitación.

\section{INTRODUCCIÓN}

El objetivo principal de la terapia endodóntica es la erradicación de bacterias del sistema de canales radiculares (Ricucci \& Siqueria Jr., 2010), ya que los microorganismos residuales juegan un papel clave en el desarrollo y perpetuación de las infecciones endodónticas. Para disminuir la carga bacteriana y lograr un mejor desbridamiento, es necesario investigar las técnicas de instrumentación y los protocolos de irrigación. Una de las principales limitaciones de la irrigación es la ausencia de un flujo turbulento, lo que dificulta la penetración del irrigante en gran parte del sistema de canales radiculares (Boutsioukis et al., 2009). Aunque la irrigación con jeringa convencional es ampliamente utilizada, se ha demostrado que el irrigante no llega a más de $2 \mathrm{~mm}$ de la punta de la aguja, lo que significa que el irrigante a menudo no alcanza la región apical del canal (Boutsioukis et al., 2010). Esto facilita la persistencia de biopelículas bacterianas y la supervivencia de una cantidad significativa de bacterias viables, aún cuando la instrumentación biomecánica se considere finalizada (Falk \& Sedgley, 2005).

\footnotetext{
${ }^{1}$ Laboratorio de Investigación en Endodoncia, Centro de investigación en Ciencias Odontológicas (CICO), Departamento integral adultos Facultad de Odontología, Universidad de La Frontera, Temuco, Chile.

2 Departamento de Odontología, Facultad de Medicina, Universidad de Barcelona, Barcelona, España.

${ }^{3}$ Laboratorio de Microbiología Molecular y Antimicrobianos, Departamento de Patología y Terapéutica Experimental, Facultad de Medicina, Universidad de Barcelona, Barcelona, España.
} 
Según Vertucci (2005), la naturaleza del sistema de canales no es uniforme y hay una alta prevalencia de canales accesorios, istmos, anastomosis y deltas apicales, que no pueden ser instrumentados. La literatura es clara en demostrar que hasta un $35 \%$ o más del sistema de canales radiculares permanece intacto luego de ser tratado con técnicas mecanizadas de instrumentación (Peters et al., 2001). Como consecuencia, restos de tejidos blandos y microorganismos (en forma planctónica o como biopelícula), pueden permanecer en el interior del sistema de canales radiculares. Esto ha sido documentado en estudios a través de cortes transversales radiculares (Rödig et al., 2002) o de microtomografía computarizada (Siqueira Jr. et al., 2020). Cabe señalar que incluso dientes uniradiculares presentan proporciones significativas de las superficies del canal sin instrumentar (Mannan et al., 2001). Dadas las limitaciones mecánicas y morfológicas descritas, para alcanzar zonas anatómicas complejas es escencial crear un flujo turbulento del irrigante.

En comparación con los métodos tradicionales, la activación del irrigante permite una desinfección más efectiva del sistema de canales radiculares (Betancourt et al., 2020). Si bien los sistemas ultrasónicos han sido el foco principal de estudio, la literatura ha respaldado recientemente el uso del láser como sistema de agitación/activación .La tecnología láser ha sido incorporada en la última década con el objetivo de mejorar la descontaminación y limpieza del sistema de canales radiculares. Existen dos enfoques para su aplicación clínica. El primero introduciendo la fibra láser en el canal radicular en seco, exponiendo la pared del canal a la luz láser con un movimiento en espiral; el segundo transmitiendo la energía a una solución irrigante (irrigación activada por láser / LAI).La interacción láser-objetivo es diferente en ambos casos. Una exposición directa del sustrato a la luz láser busca una acción mediante un efecto térmico directo, mientras que la activación y agitación del irrigante permite una acción indirecta (de Moore \& Meire, 2014).

La técnica LAI ha sido propuesta como método de desinfección complementario a la terapia quimiomecánica. El acrónimo LAI proviene del inglés "laseractivated irrigation". Hemos demostrado que su acción bactericida y de remoción de detritus es más eficiente que la irrigación convencional y que la irrigación ultrasónica pasiva (PUI) (Betancourt et al., 2019a, 2020). La mayor parte de los estudios han utilizado láseres que operan en la región del infrarrojo medio, donde la absorción por el agua es mayor, como el láser Er: YAG (longitud de onda de $2940 \mathrm{~nm}$ ) y el láser Er, Cr: YSGG (2780 nm) (George et al., 2008). La alta absorción genera efectos fotoacústicos y fotomecánicos en el fluido a medida que se crean burbujas de vapor. El mecanismo de acción de LAl ha sido descrito por Blanken \& Verdaasdonk (2007), Blanken et al. (2009), de Groot et al. (2009), Matsumoto et al. (2011), y Gregorcic et al. (2012) basándose en obtención de imágenes de alta velocidad. Los registros visuales demostraron que se da una vaporización a alta presión debido al calentamiento instantáneo de la solución. El rápido aumento de la temperatura da paso a la formación de burbujas de vapor, que se expanden para luego implosionar,fenómeno conocido como cavitación (Prosperetti, 2004). Divito et al. (2012) sugirieron que la energía liberada por la implosión de las burbujas de vapor tiene la capacidad de eliminar la capa de frotis del canal radicular y alterar las biopelículas bacterianas, produciendo alteraciones morfológicas en las membranas celulares. Estas observaciones fueron corroboradas recientemente por nuestros estudios a través de microscopía de fuerza atómica (Betancourt et al., 2019a, 2020) y microscopía electrónica de barrido (Betancourt et al., 2019b, 2020). Por otro lado, el aumento en la reactividad de la solución permite que ésta pueda fluir hacia el interior de la compleja red tridimensional del sistema de canales radiculares. Esto es de particular importancia, ya que permitiría el arribo de la solución antiscéptica a zonas morfológicas de difícil acceso (Betancourt et al., 2020). Una de las principales ventajas del LAl es posicionar la fibra láser en la entrada del canal radicular durante la activación, minimizando los efectos térmicos secundarios y reduciendo la posibilidad de extrusion apical.

El hipoclorito de sodio $(\mathrm{NaOCl})$ ha sido considerado durante largo tiempo la principal solución desinfectante en procedimientos endodónticos. Se utiliza en concentraciones que van del 0,5 al $6 \%$, presentando diferentes grados de efectividad. Dado que el $\mathrm{NaOCl}$ no es selectivo, puede dañar las células humanas, la dentina o incluso los tejidos periodontales (Zhender, 2006).

En este contexto, todavía existe controversia sobre la concentración de la solución que ofrece mayor seguridad para los tejidos del paciente sin perder la eficacia para erradicar microorganismos. La reducción de la concentración de $\mathrm{NaOCl}$ reduce la citotoxicidad del irrigante, pero desafortunadamente también reduce sus propiedades bactericidas (Radcliffe et al., 2004). Se ha mostrado que LAl mejora significativamente la eficacia antimicrobiana del $\mathrm{NaOCl}$ 
en la desinfección del canal radicular (Macedo et al., 2010). El aumento del movimiento de $\mathrm{NaOCl}$ activado incrementa su temperatura y favorece el contacto entre las moléculas de cloro activo y la materia orgánica, mejorando la eficacia química del irrigante (Macedo et al.). Por otro lado, las ondas de choque expansivas liberadas por la implosión de las burbujas de vapor,contribuyen al efecto fotomecánico, al facilitar el acceso del irrigante al tercio apical radicular y a los túbulos dentinarios (Matsumoto et al.). Sin embargo, poco se sabe sobre la efectividad antibacteriana de bajas concentraciones de $\mathrm{NaOCl}$ activado por láser,debido a que la mayoría de los estudios emplean altas concentraciones (Cheng et al., 2017a,b).

Cavitación: formación y colapso de burbujas de vapor. Cavitación se define como el crecimento y colapso rápido de una burbuja en un líquido (Prosperetti). La formación de burbujas de vapor inducidas por láser dependen en gran medida de las características del láser, como la longitud de onda, la densidad de energía, duración del pulso y la geometría de la punta láser.Una vez que la energía del láser es absorbida por la solución, hay un calentamiento instantáneo hasta el punto de ebullición, lo que resulta en la formación de una burbuja de vapor de cavitación inicial. Mientras dura la emisión láser, el rayo atraviesa la burbuja evaporando el agua, permitiendo que siga aumentando de volumen. Este fenómeno fue descrito por van Leeuwen et al. (1991) y se conoce como "efecto Moisés". Una vez que se detiene la emisión del láser, la burbuja colapsa, dando inicio a la aparición de pequeñas burbujas de cavitación secundaria, lo que induce un flujo turbulento. Este flujo genera fuerzas de cizaIla, que inicialmente se desplazan a una velocidad supersónica (ondas de choque) y posteriormente a una velocidad menor (ondas acústicas) (Matsumoto et al.). Las fuerzas liberadas son mayores dentro del canal radicular que en un espacio abierto (Matsumoto et al.). Esto se debe a que la burbuja mantiene una presión muy alta en el interior del canal, limitada por delante por el agua que la rodea, por detrás por la fibra del láser y en los lados por las paredes dentinarias. Estos fenómenos físicos pueden mejorar la eliminación de la capa de residuos y biopelículas bacterianas.

Activación láser del $\mathrm{NaOCl}$ : Fase de activación y reposo. En el interior del sistema de canales radiculares, el $\mathrm{NaOCl}$ reacciona con la materia orgánica, como el tejido pulpar, microorganismos y compuestos que forman parte de la pared dentinaria (Moorer \& Wesselink, 1982). El resultado de esta reacción es la pérdida de cloro disponible $(\Delta[\mathrm{NaOCl}])$, lo que resulta- rá en una disminución de su eficacia antimicrobiana. La tasa promedio de consumo de cloro se define como la tasa de reacción (RR) y se puede determinar mediante el cociente entre la diferencia en la concentración de $\mathrm{NaOCl}$ antes y después del tiempo de exposición $(\Delta[\mathrm{NaOCl}])$ y el tiempo de exposición total $(\Delta t)$ $(\mathrm{RR}=\Delta[\mathrm{NaOCl}] / \Delta \mathrm{t})($ Macedo et al.). Las moléculas de los irrigantes activados por láser se movilizan mediante convección sostenida por microcorriente acústica. La mayor velocidad de movimiento de las moléculas activadas por láser, mejora la eficacia del $\mathrm{NaOCl}$, aumentando el contacto del cloro libre con materia orgánica y/o biopelículas bacterianas en su interior. Por otra parte, el incremento de la turbulencia eleva la temperatura del $\mathrm{NaOCl}$, favoreciendo su reactividad y efecto bactericida (Sirtes et al., 2005). Kuhn et al. (2013) investigaron in vitro el efecto de disolución de tejidos blandos del $\mathrm{NaOCl}$ activado con láser Er: YAG. El estudio concluyó que la activación láser del $\mathrm{NaOCl}$, utilizando una potencia de $0,2 \mathrm{~W}$, conduce a una disolución efectiva de los tejidos blandos. Antes de realizar el recambio del $\mathrm{NaOCl}$, es fundamental tener en cuenta la fase de reposo. Macedo et al. observaron que durante un intervalo de 3 minutos entre los ciclos de activación, el consumo de cloro disponible aumentó significativamente. Por tanto, la inclusión de una fase de reposo luego de la activación láser, permite que el $\mathrm{NaOCl}$ reaccione aún más (Betancourt et al., 2019b).

Modelos de estudio bacterianos empleados en LAI. Enterococcus faecalis ha sido la especie bacteriana empleada con mayor frecuencia en el estudio de LAl. Una gran parte de las investigaciones que exploran la efectividad de agentes antimicrobianos en endodoncia utilizan bacterias en estado planctónico (Schoop et al., 2007). Sin embargo, las infecciones bucales son causadas principalmente por bacterias sésiles y las infecciones endodónticas no son una excepción. Un factor relevante de la investigación realizada en biopelículas es la edad, ya que las bacterias reaccionan de manera diferente no solo cuando viven como planctónicas o sésiles, sino que también pueden observarse diferencias entre las células que viven en biopeliculas jóvenes y maduras. La edad de las biopelículas utilizadas en biología experimental es con frecuencia un tema de discusión. La mayoría de las investigaciones se realizan con biopelículas de 24h (Betancourt et al., 2019a) o 48h (Yavari et al., 2010), mientras que en la práctica clínica es muy probable que tengamos que combatir biopelículas mucho más antiguas (hasta 10 días o más) (Licata et al., 2015; Sans-Serramitjana et al., 2016; Betancourt et al., 2020). Las incubaciones bacterianas de más tiempo ofrecen características más 
relevantes, debido a la formación de biopelículas maduras. Se han propuesto diversos modelos de infección con el fin de dilucidar las perspectivas en el uso del láser para lograr la desinfección del canal; esto incluye dientes humanos utilizados ex vivo (Christo et al., 2016; Betancourt et al., 2019b, 2020), canales radiculares artificiales (de Meyer et al., 2017; Betancourt et al., 2019a; Swimberghe et al., 2019), cortes de dentina de dientes bovinos (Franzen et al., 2011) y cortes de dentina de raíz humana (Juric et al., 2016). En todos los casos, los irrigantes endodónticos presentan dificultades para llegar al extremo distal de los canales.

\section{Interacción entre láser y baja concentración de} hipoclorito de sodio: un efecto sinérgico. Como hemos comentado, un desafío en la endodoncia es encontrar alternativas para reducir la toxicidad del $\mathrm{NaOCl}$ sin perder la actividad antibacteriana. Así, el estudio de eventuales efectos sinérgicos entre láser y bajas concentraciones de $\mathrm{NaOCl}$ se ha convertido en un campo de gran interés. Betancourt et al. (2019a) observaron a través de un modelo in vitro, que la acción antibacteriana del $\mathrm{NaOCl}$ al $0,5 \%$ ante biofilm de $E$. faecalis aumentaba al activarlo mediante láser de Er,Cr:YSGG. Mediante microscopía de fuerza atómica corroboramos los resultados microbiológicos, observando una pérdida de integridad en las células bacterianas tratadas por LAI (Fig. 1). Jaramillo et al.
(2016) reportaron que la activación de $\mathrm{NaOCl}$ al 0,5\% tamponado mejoró su capacidad antibacteriana contra biofilm de $E$. faecalis de 4 semanas de formación en dientes extraídos. Por otro lado, en un estudio realizado en dientes extraídos, Betancourt et al. (2019b) observaron que la acción bactericida del $\mathrm{NaOCl}$ al 0,5 $\%$ activado por láser de $\mathrm{Er}, \mathrm{Cr}$ :YSGG fue semejante a la alcanzada por el $\mathrm{NaOCl}$ al 2,5\%, lo que significa 5 veces su concentración inicial. Sin embargo, no todos los artículos están de acuerdo. Christo et al. no lograron demostrar una mejora del $0,5 \%$ de $\mathrm{NaOCl}$ en biopelículas idénticas. Esto puede deberse a que el estudio fue realizado con un láser Er, Cr: YSGG a baja potencia $(0,5 \mathrm{~W})$.

\section{Posición de la fibra láser y su relación con} extrusión del irrigante. La posibilidad de extrusión accidental del irrigante más allá de la constricción apical debe tenerse en cuenta. Hasta ahora, diversos protocolos de LAI han propuesto posicionar la fibra a diferentes niveles, realizando la activación a $5 \mathrm{~mm}$ del ápice (Deleu et al., 2015), a $1 \mathrm{~mm}$ de la longitud de trabajo (de Groot et al.), hasta $4 \mathrm{~mm}$ desde la entrada del canal (Seet et al., 2012), a la entrada del canal (Betancourt et al., 2019b, 2020) y últimamente en la cámara pulpar (Yang et al., 2020). Peeters \& De Moore (2015) demostraron que la probabilidad de extrusión es mayor cuanto más cerca del ápice radicular se active la fibra óptica. Por lo tanto, se debe tener en consi-
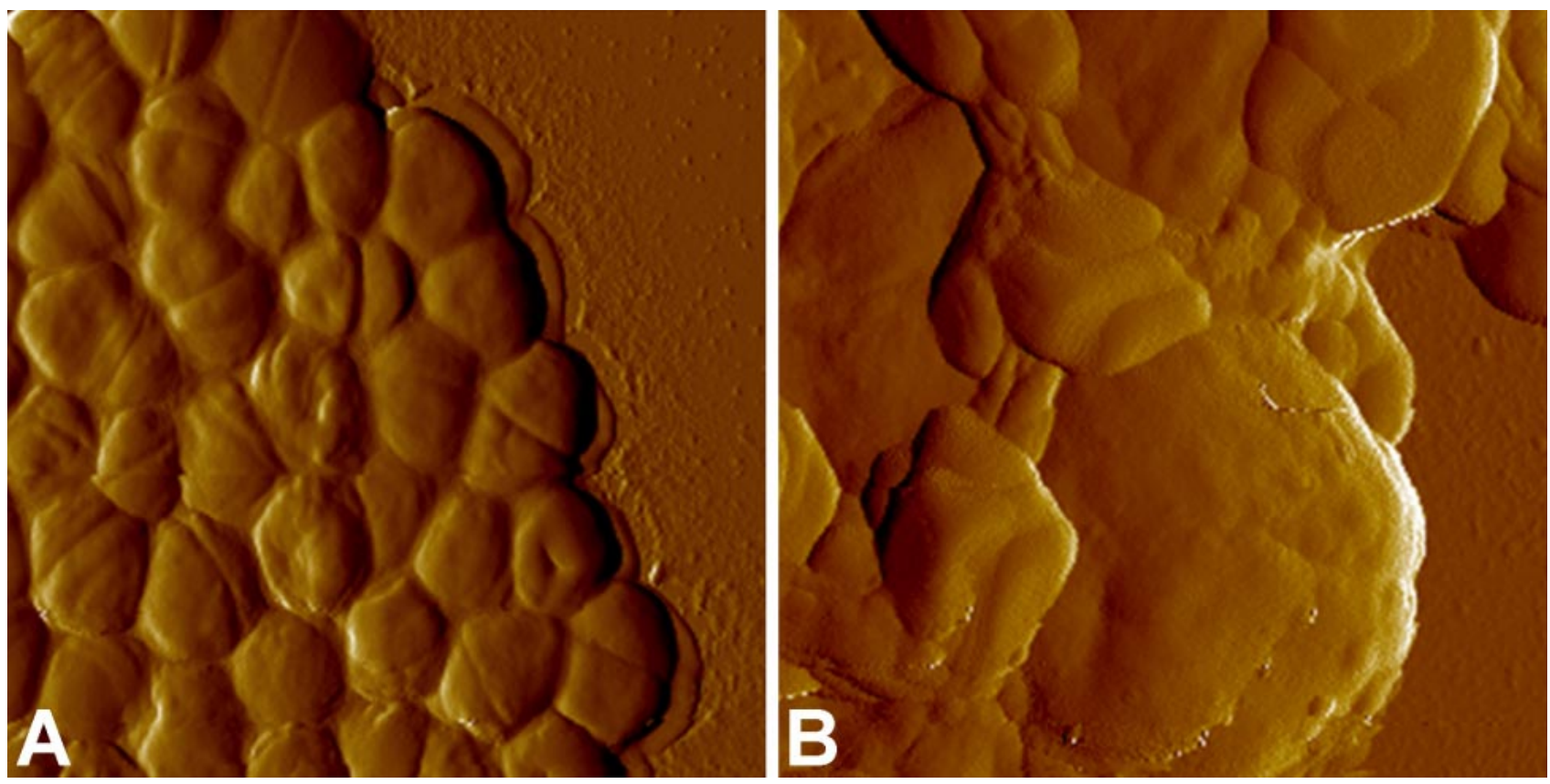

Fig. 1. Imágenes de microscopía de fuerza atómica de biopelícula de E. faecalis de 10 días de formación. A) Biopelícula sin tratar. B) Biopelícula tratada por hipoclorito de sodio al 0,5\% activado por láser Er,Cr:YSGG. Se aprecia la pérdida de integridad de la morfología de las células tratadas, lo que es una muestra dela eficacia del tratamiento. 
deración que al activar el irrigante a nivel de la cámara pulpar y de la entrada del canal, se disminuirá considerablemente la probabilidad de extrusión del irrigante. Recientemente, Peeters et al. (2018) estudiaron el nivel de extrusión de medio de contraste radiopaco en 20 dientes con ápice abierto utilizando Er, Cr: YSGG LAI (1W, $35 \mathrm{~Hz})$. El resultado fue la ausencia total de medio de contraste en todos los casos, demostrando la seguridad de la técnica. Sin embargo, para evitar efectos no deseados, se debe tener extrema precaución cuando se utiliza LAI, particularmente en la proximidad de la constricción apical. Otro factor relevante a considerar es la temperatura. Se ha señalado que un aumento de la temperatura provocada por el láser puede generar efectos indeseables en la dentina, como grietas, pequeñas fisuras o incluso carbonización (Yamada et al., 2004). En experimentos llevados a cabo por nuestro grupo de investigación, utilizamos la fibra láser lejos del ápice dentario, impidiendo en cualquier caso el contacto de la fibra y las paredes del canal. De ésta forma se consiguió proteger la estructura dentinaria frente a posibles daños térmicos. La eficacia de este procedimiento fue confirmado por SEM, que reveló dentina intacta después del tratamiento (Betancourt et al., 2019b, 2020). La posición más coronal de la fibra láser ha demostrado ser efectiva tanto en la penetración del irrigante al interior de los túbulos dentinarios (Fig. 2), como en la remoción del barro dentinario. Esto se ha puesto de manifiesto observando túbulos dentinarios abiertos tras la terapia (Fig. 3). Teniendo en cuenta que en canales estrechos los sistemas sónicos y ultrasónicos están limitados por el contacto con la pared, LAI podría ser una herramienta adecuada para limpiar el canal radicular de manera eficiente, especialmente en canales con curvaturas pronunciadas (Cheng et al., 2017a). El protocolo que hemos propuesto utilizando un láser pulsado Er, Cr: YSGG (2780nm), consiste en emplear $1 \mathrm{~W}$ de potencia, $10 \mathrm{~Hz}$ de frecuencia de repetición, $100 \mathrm{~mJ}$ de energía por pulso. Realizar un ciclo de activación de EDTA al $17 \%$ durante 30 s, 2 ciclos de activación por 30 s de $\mathrm{NaOCl}$ al $0,5 \%$, dejando 30 s de reposo entre cada ciclo, posicionando la fibra óptica a la entrada del canal radicular durante todo el proceso. Finalmente irrigar con solución salina antes de la fase de obturación (Betancourt et al., 2019b, 2020).

Evolución de LAl: Pips y Sweeps PIPS (Photon Induced Photo-acoustic Streaming) y Sweeps (Shock Wave Enhanced Emission Photoacoustic Streaming) son los últimos protocolos basados en activación láser del irrigante. Ambas técnicas derivan del mecanismo de acción de LAl y emplean un láser Er:YAG (2940nm). Entre las diferencias con la técnica LAI
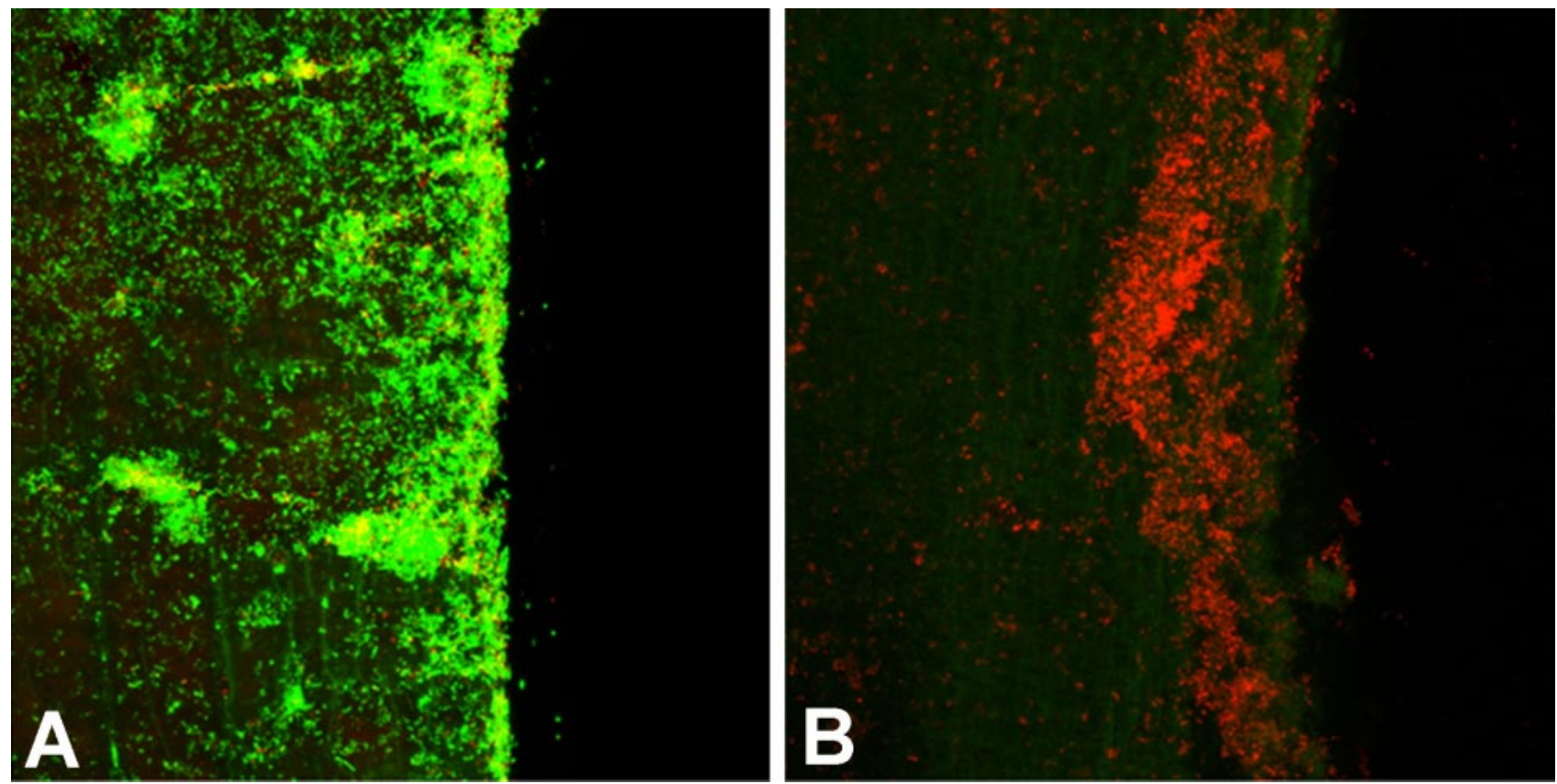

Fig. 2. Imagen de biopelícula de E. faecalis de 10 días de crecimiento, a través de microscopía confocal láser de barrido. En verde se aprecian las bacterias vivas, en rojo las bacterias muertas. A) Biopelícula sin tratar. B) Biopelícula tratada con 0,5 $\%$ de $\mathrm{NaOCl}$ activado por láser Er,Cr:YSGG. EI NaOCl activado fue impulsado al interior de los túbulos dentinarios. Barra de escala: $50 \mu \mathrm{m}$. 

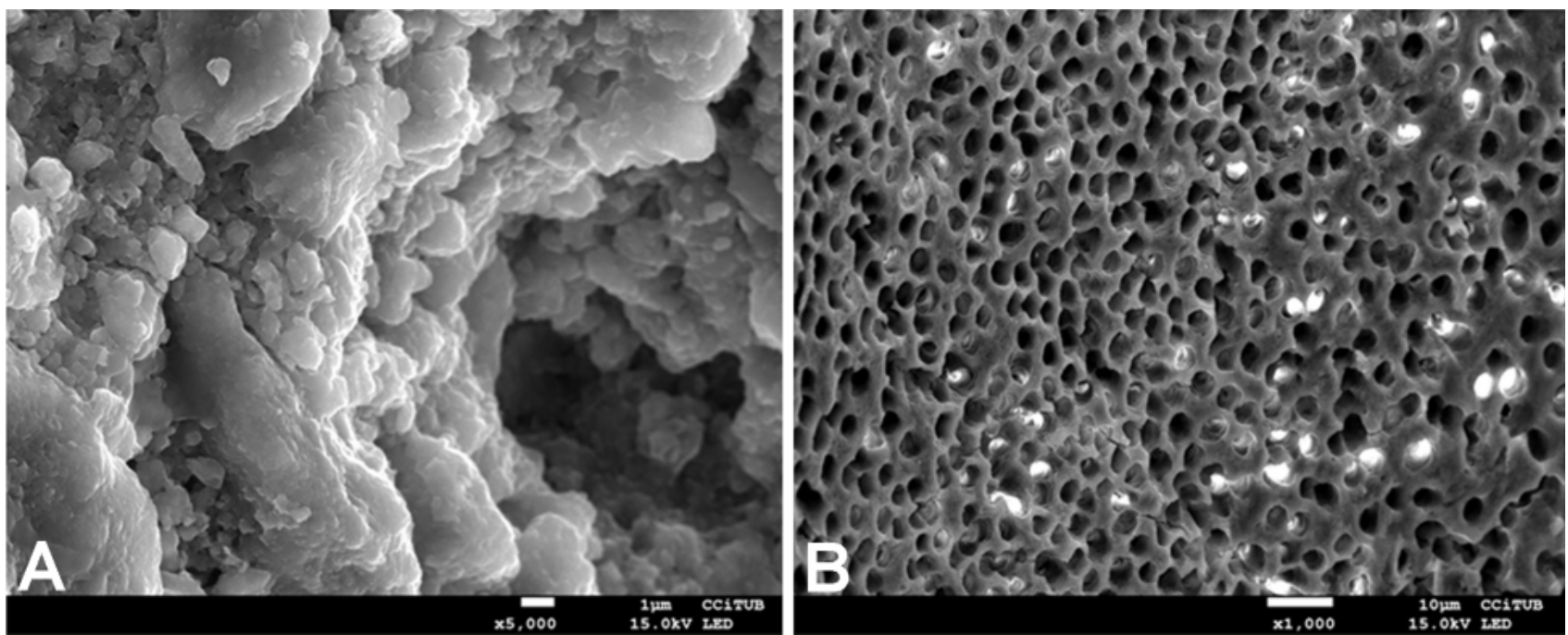

Fig. 3. Biopelícula de E. faecalis, 10 días de formación, captada por microscopía electrónica de barrido. A) Biopelícula sin tratar. B) Superficie dentinaria luego de ser tratada con hipoclorito de sodio al 0,5\% activado por láser Er,Cr:YSGG. Se aprecia un alto grado de limpieza, tanto en la superficie como en el interior de los túbulos dentinarios luego del tratamiento.

tradicional destaca la utilización de pulsos de energía más bajos y una duración de pulso más corto. Además, la posición de trabajo de la fibra láseres a nivel de la cámara pulpar, y no en el canal como LAI.La activación del láser en la cámara pulpar, permite tratar simultánemanete canales de dientes multirradiculares, evitar efectos térmicos secundarios y reducir la posibilidad de extrusión del irrigante a los tejidos pericapicales.

La irrigación SSP (Super Short Pulse) también conocida como PIPS, es un protocolo clínico basado en la cavitación (Koch et al., 2016). El láser utiliza una energía de pulso baja $(20 \mathrm{~mJ})$ y de corta duración (50 mseg) (Divito et al., 2011). La activación PIPS ha demostrado una buena eficacia antimicrobiana (Peters et al., 2011; Pedullà et al., 2012), así como una mayor disolución de los tejidos pulpares (Guneser et al., 2015). Algunos estudios han reportado un incremento en la eliminación de la capa de frotis (Ekim \& Erdemir, 2015; Keles et al., 2016), mientras que otros informan una mejora mínima (Nasher et al., 2016). El protocolo de irrigación final utiliza EDTA al $17 \%$ activado durante 30 s; después de 60 s de reposo, se activa agua destilada estéril durante 30 s y luego, tres ciclos de activación de $\mathrm{NaOCl}$ al 5 $\%$ o $6 \%$ durante 30 s; cada ciclo tiene una fase de reposo de 30s. Finalmente, se activa solución salina durante 30s para inactivar el oxígeno presente. SWEEPS representa la evolución tecnológica de PIPS (Lukac" et al., 2017). La modalidad de pulso supercorto (50 mseg; el mismo en cuanto a PIPS) se acompaña de la modalidad de pulso único ultra-corto (25 mseg) que permite mejor modulación de la energía emitida, manteniendo la misma potencia máxima (es decir, $400 \mathrm{~W}$ utilizando $10 \mathrm{~mJ}$ ) o una potencia pico más potente $(800 \mathrm{~W})$ utilizando la misma energía que PIPS (20 mJ).Al utilizar esta nueva modalidad, se logra la formación y colapso de burbujas de vapor en el irrigante, lo que lleva a la emisión de ondas de choque, facilitando la limpieza incluso en canales radiculares estrechos (Lukac \& Jezersek, 2018). El protocolo final de irrigación implica dos ciclos de activación de EDTA al $17 \%$ (30 s), seguidos de activación desolución salina(30 s), luego tres ciclos de activación de $\mathrm{NaOCl}$ al $5 \%$ (30 s) y un tiempo de reposo de al menos 30s entre cada ciclo. En un estudio reciente, SWEEPS fue asociado con un aumento significativo en el grado de limpieza, principalmente de zonas de itsmos en raíces mesiales (Yang et al.). Sin embargo al ser comparado con PIPS, SWEEPS no evidenció una mejora en la penetración del irrigante en el canal radicular (Galler et al., 2019). Se requieren nuevos estudios para determinar el efecto antibacteriano real y el efecto de remoción de barro dentinario del interior del canal radicular.

\section{LIMITACIONES}

La amplia variabilidad en los protocolos y parámetros publicados, dificultan la elección de la técnica a emplear. Por otro lado,la biopelícula 
monoespecie de $E$. faecalis ha sido por lejos el modelo bacteriano más empleado para determinar el poder antibacteriano de LAI. Se ha establecido que las infecciones endodónticas persistentes se componen mayoritariamente de comunidades polimicrobianas, por lo que sería adecuado la incorporación de biopelículas multiespecies al diseño metodológico de nuevos estudios. Finalmente, la mayoría de las investigaciones han utilizado modelos de experimentación in vitro o ex vivo. Es necesario la estandarización de protocolos y el desarrollo de ensayos clínicos para determinar la efectividad real de LAl.

BETANCOURT, P.; ARNABAT-DOMÍNGUEZ, J. \& VIÑAS, M. Laser activated irrigation in endodontics. Int. J. Odontostomat., 15(3):773-781, 2021.

ABSTRACT: The goal of endodontics is to prevent or cure apical periodontitis. Therefore, microorganisms that have colonized the root canal system must be eliminated to promote regeneration and healing. Unfortunately, the existence of accessory canals, anastomoses, isthmus, as well as apical ramifications, form a complex three-dimensional network inside the root, which makes difficult the total elimination of bacteria and detritus. Conventional endodontic therapy has several limitations. Endodontic instruments have been shown to leave $35 \%$ or more of the dentin surface untreated. On the other hand, the inability of generating a turbulent flow, prevents the irrigant from reaching areas that are difficult to access. This facilitates the persistence of bacterial biofilms and the survival of a significant number of viable bacteria. Laser-activated Irrigation (LAI) has been proposed as a co-adjuvant to chemo-mechanical therapy to improve cleaning and disinfection. Er, Cr: YSGG (2780nm) and Er: YAG (2940nm) lasers are the most widely used. These two wavelengths are capable of being widely absorbed by different irrigating solutions, taking into account safe use and respecting accepted clinical parameters. The deep absorption of laser energy generates steam bubbles inside the fluid, which release shear forces when imploding. This phenomenon, called cavitation, is responsible for greater cleaning and disinfection within the root canal system, even in areas difficult to access. Several studies have demonstrated the antibacterial effectiveness of LAl through microbiological methods and microscopic techniques. Recently, it has been reported that LAl would have the ability to increase the antibacterial capacity of low concentration of sodium hypochlorite (a good disinfectant but extremely toxic), which would allow working with concentrations that are safer for the patient.

KEY WORDS: laser, irrigation, endodontics, sodium hypochlorite, cavitation.

\section{REFERENCIAS BIBLIOGRÁFICAS}

Betancourt, P.; Merlos, A.; Sierra, J. M.; Arnabat-Dominguez, J. \& Viñas, M. Er,Cr:YSGG laser-activated irrigation and passive ultrasonic irrigation: comparison of two strategies for root canal disinfection. Photobiomodul. Photomed. Laser. Surg., 38(2):91-7, 2020.

Betancourt, P.; Merlos, A.; Sierra, J. M.; Camps-Font, O.; ArnabatDominguez, J. \& Viñas, M. Effectiveness of low concentration of sodium hypochlorite activated by Er,Cr:YSGG laser against Enterococcus faecalis biofilm. Lasers Med. Sci., 34(2):247$54,2019 a$.

Betancourt, P.; Sierra, J. M.; Camps-Font, O.; Arnabat-Domínguez, J. \& Viñas, M. Er,Cr:YSGG laser-activation enhances antimicrobial and antibiofilm action of low concentrations of sodium hypochlorite in root canals. Antibiotics (Basel), 8(4):232, $2019 \mathrm{~b}$.

Blanken, J. \& Verdaasdonk, R. Cavitation as a working mechanism of the Er, Cr: YSGG laser in endodontics: A visualization study. J. Oral Laser Appl., 7(2):97-106, 2007.

Blanken, J.; De Moor, R. J. G.; Meire, M. \& Verdaasdonk, R. Laser induced explosive vapor and cavitation resulting in effective irrigation of the root canal. Part 1: A visualization study. Lasers Surg. Med., 41(7):514-9, 2009.

Boutsioukis, C.; Lambrianidis, T. \& Kastrinakis, E. Irrigant flow within a prepared root canal using various flow rates: a computational fluid dynamics study. Int. Endod. J., 42(2):14455, 2009.

Boutsioukis, C.; Lambrianidis, T.; Verhaagen B, Versluis M.; Kastrinakis E.; Wesselink P. R. \& van der Sluis, L. W. M. The effect of needle-insertion depth on the irrigant flow in the root canal: evaluation using an unsteady computational fluid dynamics model. J. Endod., 36(10):1664-8, 2010.

Cheng, X.; Tian, T.; Tian, Y.; Xiang, D.; Qiu, J.; Liu, X. \& Yu, Q. Erbium:Yttrium aluminum garnet laser- activated sodium hypochlorite irrigation: a promising procedure for minimally invasive endodontics. Photomed. Laser Surg., 35(12):695-701, 2017a.

Cheng, X.; Xiang, D.; He, W.; Qiu, J.; Han, B.; Yu, Q. \& Tian, Y. Bactericidal effect of Er:YAG laser-activated sodium hypochlorite irrigation against biofilms of Enterococcus faecalis isolate from canal of root-filled teeth with periapical lesions. Photomed. Laser Surg., 35(7):386-92, 2017b.

Christo, J. E.; Zilm, P. S.; Sullivan, T. \& Cathro, P. R. Efficacy of low concentrations of sodium hypochlorite and low-powered $\mathrm{Er}, \mathrm{Cr}$ : YSGG laser activated irrigation against an Enterococcus faecalis biofilm. Int.Endod. J., 49(3):279-86, 2016.

de Groot, S. D.; Verhaagen, B.; Versluis, M.; Wu, M. K.; Wesselink, P. R. \& van der Sluis, L. W. M. Laser-activated irrigation within root canals: cleaning efficacy and flow visualization. Int. Endod. J., 42(12):1077-83, 2009.

De Meyer, S.; Meire, M. A.; Coenye, T. \& De Moor, R. J. Effect of laser- activated irrigation on biofilms in artificial root canals. Int. Endo. J., 50(5):472-9, 2017.

De Moor, R. J. \& Meire, M. High-power lasers in endodontics Fiber placement for laser-enhanced endodontics: In the canal or at the orifice? J. Laser Health, 1:20-8, 2014.

Deleu, E.; Meire, M. A. \& De Moor, R. J. Efficacy of laser-based irrigant activation methods in removing debris from simulated root canal irregularities. Lasers. Med. Sci., 30(2):831-5, 2015.

Divito, E. E.; Colonna, M. P. \& Olivi, G. The Photoacoustic Efficacy of an Er:YAG laser with radial and stripped tips on root canal dentin walls: an SEM evaluation. J. Lasers. Dent., 19(1):15661, 2011. 
Divito, E.; Peters, O. A. \& Olivi, G. Effectiveness of the erbium:YAG laser and new design radial and stripped tips in removing the smear layer after root canal instrumentation. Lasers Med. Sci., 27(2):273-80, 2012.

Ekim, S. N. A. \& Erdemir, A. Comparison of different irrigation activation techniques on smear layer removal: an in vitro study. Microsc. Res. Tech., 78(3):230-9, 2015.

Falk, K. W. \& Sedgley, C. M. The influence of preparation size on the mechanical efficacy of root canal irrigation in vitro. J. Endod., 31(10):742-5, 2005.

Franzen, R.; Gutknecht, N.; Falken, S.; Heussen, N. \& Meister, J. Bactericidal effect of a Nd:YAG laser on Enterococcus faecalis at pulse durations of 15 and $25 \mathrm{~ms}$ in dentine depths of 500 and 1,000um. Lasers Med. Sci., 26:95-101, 2011.

Galler, K. M.; Grubmüller, V.; Schlichting, R.; Widbiller, M.; Eidt, A.; Schuller, C.; Wölflick, M.; Hiller, K. A. \& Buchalla, W. Penetration depth of irrigants into root dentine after sonic, ultrasonic and photoacoustic activation. Int. Endod. J., 52(8):1210-7, 2019.

George, R.; Meyers, I. A. \& Walsh, L. J. Laser activation of endodontic irrigants with improved conical laser fiber tips for removing smear layer in the apical third of the root canal. J. Endod., 34(12):15247, 2008.

Gregorcic, P.; Jezersek, M. \& Mozina, J.Optodynamic energyconversion efficiency during an Er:YAG-laser-pulse delivery into a liquid through different fiber-tip geometries. J. Biomed. Opt., 17(7):075006, 2012.

Guneser, M. B.; Arslan, D. \& Usumez, A. Tissue dissolution ability of sodium hypochlorite activated by photon-initiated photoacoustic streaming technique. J. Endod., 41(5):729-32, 2015.

Jaramillo, D. E.; Aguilar, E.; Arias, A.; Ordinola-Zapata, R.; Aprecio, R. M. \& Ibarrola, J. L. Root canal disinfection comparing conventional irrigation vs photon-induced photoacoustic streaming (PIPS) using a buffered $0.5 \%$ sodium hypochlorite solution. Evid. Based Endod., 1:6, 2016.

Juric, I. B.; Plecko, V.; Anic, I.; Plesko, S.; Jakovljevic, S.; Rocca, J. P.\& Medioni, E. Antimicrobial efficacy of photodynamic therapy, $\mathrm{Nd}$ :YAG laser and QMIX solution against Enterococcus faecalis biofilm. Photodiagnosis Photodyn. Ther., 13:238-43, 2016.

Keles, A.; Kamalak, A.; Keskin, C.; Akçay, M. \& Uzun, I. The efficacy of laser, ultrasound and self-adjustable file in removing smear layer debris from oval root canals following retreatment: $A$ scanning electron microscopy study. Aust. Endod. J., 42(3):10411, 2016.

Koch, J. D.; Jaramillo, D. E.; DiVito, E. \& Peters, O. A. Irrigant flow during Photon-induced Photoacoustic Streaming (PIPS) using Particle Image Velocimetry (PIV). Clin. Oral Investig., 20(2):3816, 2016.

Kuhn, K.; Rudolph, H.; Luthardt, R. G.; Stock, K.; Diebolder, R. \& Hibst, R. Er:YAG laser activation of sodium hypochlorite for root canal soft tissue dissolution. Lasers Surg. Med., 45(5):33944, 2013.

Licata, M. E.; Albanese, A.; Campisi, G.; Geraci, D. M.; Russo, R. \& Gallina, G. Effectiveness of a new method of disinfecting the root canal, using Er, Cr:YSGG laser to kill Enterococcus faecalis in an infected tooth model. Lasers Med. Sci., 30(2):707-12, 2015.

Lukac, N. \& Jezersek, M. Amplification of pressure waves in laserassisted endodontics with synchronized delivery of Er:YAG laser pulses. Lasers Med. Sci., 33(4):823-33, 2018.

Lukac", N.; Tasic Muc, B.; Jezeršek, M. \& Lukac, M. Photoacousticendodontics using the novel SWEEPS Er: YAG laser modality. J. Laser Health Acad., 2017(1):1-7, 2017.

Macedo, R. G.; Wesselink, P. R.; Zaccheo, F.; Fanali, D. \& van der Sluis, L. W. M. Reaction rate of $\mathrm{NaOCl}$ in contact with bovine dentine:effect of activation, exposure time, concentration and pH. Int. Endod. J., 43(12):1108-15, 2010.
Mannan, G.; Smallwood, E. \& Gulabivala, K. The influence of access cavity design on filing of canals in anterior teeth. Int. Endod. J., 34(3):176-83, 2001.

Matsumoto, H.; Yoshimine, Y. \& Akamine, A. Visualization of irrigant flow and cavitation induced by Er:YAG laser within a root canal model. J. Endod., 37(6):839-43, 2011.

Moorer, W. R. \& Wesselink, P. R. Factors promoting the tissue dissolving capability of sodium hypochlorite. Int. Endod. J., 15(4):187-96, 1982.

Nasher, R.; Franzen, R. \& Gutknecht, N. The effectiveness of the Erbium:Yttrium aluminum garnet PIPS technique in comparison to different chemical solutions in removing the endodontic smear layer-an in vitro profilometric study. Lasers Med. Sci., 31(9):1871-82, 2016.

Pedullà, E.; Genovese, C.; Campagna, E. \& Tempera, R. E. Decontamination efficacy of photon-initiated photoacoustic streaming (PIPS) of irrigants using low-energy laser settings: an ex vivo study. Int. Endod. J., 45(9):865-70, 2012.

Peeters, H. H. \& De Moor, R. J. G. Measurement of pressure changes during laser-activated irrigant by an erbium, chromium: yttrium, scandium, gallium, garnet laser. Lasers Med., Sci., 30(5):1449-55, 2015.

Peeters, H. H.; Suardita, K.; Mooduto, L. \& Gutknecht, N. Extrusion of irrigant in open apex teeth with periapical lesions following laser-activated irrigation and passive ultrasonic irrigation. Iran. Endod. J., 13(2):169-75, 2018.

Peters, O. A.; Bardsley, S.; Fong, J.; Pandher, G. \& Divito, E. Disinfection of root canals with photon-initiated photoacoustic streaming. J. Endod., 37(7):1008-12, 2011.

Peters, O. A.; Schönenberger, K. \& Laib, A. Effects of four Ni-Ti preparation techniques on root canal geometry assessed by micro computed tomography. Int. Endod. J., 34(3):221-30, 2001.

Prosperetti, A. Bubbles. Phys. Fluids, 16:1852-65, 2004.

Radcliffe, C. E.; Potouridou, L.; Qureshi, R.; Habahbeh, N.; Qualtrough, A.; Worthington, H. \& Drucker, D. B. Antimicrobial activity of varying concentrations of sodium hypochlorite on the endodontic microorganisms Actinomyces israelii, A. naeslundii, Candida albicans and Enterococcus faecalis. Int. Endod. J., 37(7):438-46, 2004.

Ricucci, D. \& Siqueira Jr., J. F. Biofilms and apical periodontitis: study of prevalence and association with clinical and histopathologic findings. J. Endod., 36(8):1277-88, 2010.

Rödig, T.; Hülsmann, M.; Mühge, M. \& Schäfers, F. Quality of preparation of oval distal root canals in mandibular molars using nickel-titanium instruments. Int. Endod. J., 35(11):91928, 2002.

Sans-Serramitjana, E.; Fusté, E.; Martínez-Garriga, B.; Merlos, A.; Pastor, M.; Pedraz, J. L.; Esquisabel, A.; Bachiller, D.; Vinuesa, T. \& Viñas, M. Killing effect of nanoencapsulated colistin sulfate on Pseudomonas aeruginosa from cystic fibrosis patients. J. Cyst. Fibros., 15(5):611-8, 2016.

Schoop, U.; Goharkhay, K.; Klimscha, J.; Zagler, M.; Wernisch, J.; Georgopoulos, A.; Sperr, W. \& Moritz, A. The use of the erbium, chromium:yttrium-scandium-gallium-garnet laser in endodontic treatment: the results of an in vitro study. J. Am. Dent. Assoc., 138(7):949-55, 2007.

Seet, A. N.; Zilm, P. S.; Gully, N. J. \& Cathro, P. R. Qualitative comparison of sonic or laser energisation of $4 \%$ sodium hypochlorite on an Enterococcus faecalis biofilm grown in vitro. Aust. Endod. J., 38(3):100-6, 2012.

Siqueira Jr., J. F.; Antunes, H. S.; Pérez, A. R.; Alves, F. R. F.; Mdala, I.; Silva, E. J. N. L.; Belladonna, F. G. \& Rôças, I. N. The apical root canal system of teeth with posttreatment apical periodontitis: correlating microbiologic, tomographic, and histopathologic findings. J. Endod., 46(9):1195-203, 2020. 
Sirtes, G.; Waltimo, T.; Schaetzle, M. \& Zehnder, M. The effects of temperature on sodium hypochlorite short-term stability, pulp dissolution capacity, and antimicrobial efficacy. J. Endod., 31(9):669-71, 2005.

Swimberghe, R. C. D.; De Clercq, A.; de Moor, R. J. G. \& Meire, M. A. Efficacy of sonically, ultrasonically and laser-activated irrigation in removing a biofilm-mimicking hydrogel from an isthmus model. Int. Endod. J., 52(4):515-23, 2019.

van Leeuwen, T. G.; van der Veen, M. J.; Verdaasdonk, R. M. \& Borst, C. Noncontact tissue ablation by holmium:YSGG laser pulses in blood. Lasers Surg. Med., 11:26-34, 1991.

Vertucci, F. J. Root canal morphology and its relationship to endodontic procedures. Endod. Topics, 10(1):3-29, 2005.

Yamada, M. K.; Uo, M.; Ohkawa, S.; Akasaka, T. \& Watari, F. Threedimensional topographic scanning electron microscope and Raman spectroscopic analyses of the irradiation effect on teeth by Nd:YAG, Er: YAG, and CO(2) lasers. J. Biomed. Mater. Res. B Appl. Biomater., 71(1):7-15, 2004.

Yang, Q.; Liu, M. W.; Zhu, L. X. \& Peng, B. Micro-CT study on the removal of accumulated hard-tissue debris from the root canal system of mandibular molars when using a novel laser-activated irrigation approach. Int. Endod. J., 53(4):529-38, 2020.

Yavari, H. R.; Rahimi, S.; Shahi, S.; Lotfi, M.; Barhaghi, M. H.; Fatemi, A. \& Abdolrahimi, M. Effect of Er, Cr: YSGG laser irradiation on Enterococcus faecalis in infected root canals. Photomed. Laser Surg., 28 Suppl. 1:S91-6, 2010.

Zhender, M. Root canal irrigants. J. Endod., 32(5):389-98, 2006.

\section{Dirección para correspondencia:}

Dr. Pablo Betancourt

Profesor Asistente

Centro de investigación en Ciencias Odontológicas

Facultad de Odontología

Universidad de La Frontera

Avenida Francisco Salazar 01145

Temuco

CHILE

E-mail: pablo.betancourt@ufrontera.cl

Pablo Betancourt: orcid.org/0000-0002-9903-2920

Josep Arnabat Domínguez: orcid.org/0000-0003-1653-8043

Miguel Viñas: orcid.org/0000-0003-2073-3386 